\title{
Photon reconstruction and identification in heavy ion collisions with the CMS detector
}

\author{
Yeonju Go on behalf of the CMS Collaboration ${ }^{1, a}$ \\ ${ }^{1}$ Korea University, 145 Anam-ro, Seonbuk-gu, Seoul, Republic of Korea
}

\begin{abstract}
Measurement of isolated photon gives a possibility to constrain the nuclear parton distribution functions (nPDF). The isolated photon is also the clear tag in the photon-jet channel for studying in-medium modifications of fragmentation functions of parton initiated jets. The performance of isolated photon reconstruction and identification has been studied in $\mathrm{PbPb}$ collisions at $\sqrt{s_{N N}}=5.02 \mathrm{TeV}$. Trigger, reconstruction and isolation efficiencies are measured in PYTHIA+HYDJET Monte-Carlo simulation. We describe signal template method to determine the purity of isolated photons.
\end{abstract}

\section{Introduction}

Prompt photons coming from the primary vertex of the high energy hadron collisions are unaffected by the strongly interacting medium produced in the reaction. Prompt photons are the 'clean' probes of the initial state of the collision and play key roles in better understanding the Quark-Gluon Plasma (QGP). Isolated photons include prompt photons and collinear fragmentation photons of a parton producing large transverse momentum. The performance of reconstruction and identification of isolated photons has been studied with data collected in $2015 \mathrm{PbPb}$ collisions at $\sqrt{s_{N N}}=5.02 \mathrm{TeV}$ and PYTHIA+HYDJET Monte Carlo simulation. In this analysis, isolated photon performances have been studied with respect to its transverse momentum and centrality, which is defined by the impact parameter $b$. Experimentally, centrality is expressed as a percentage of the total nuclear interaction cross-section.

\section{Efficiency}

\subsection{Trigger Efficiency}

Figure 1 shows trigger efficiency for different photon threshold (left) and reconstructed photon spectra coming from all the photon trigger combination without isolation condition (right). All trigger efficiencies are over $99 \%$ and have sharp turn-on feature for each threshold.

ae-mail: ygo@cern.ch 

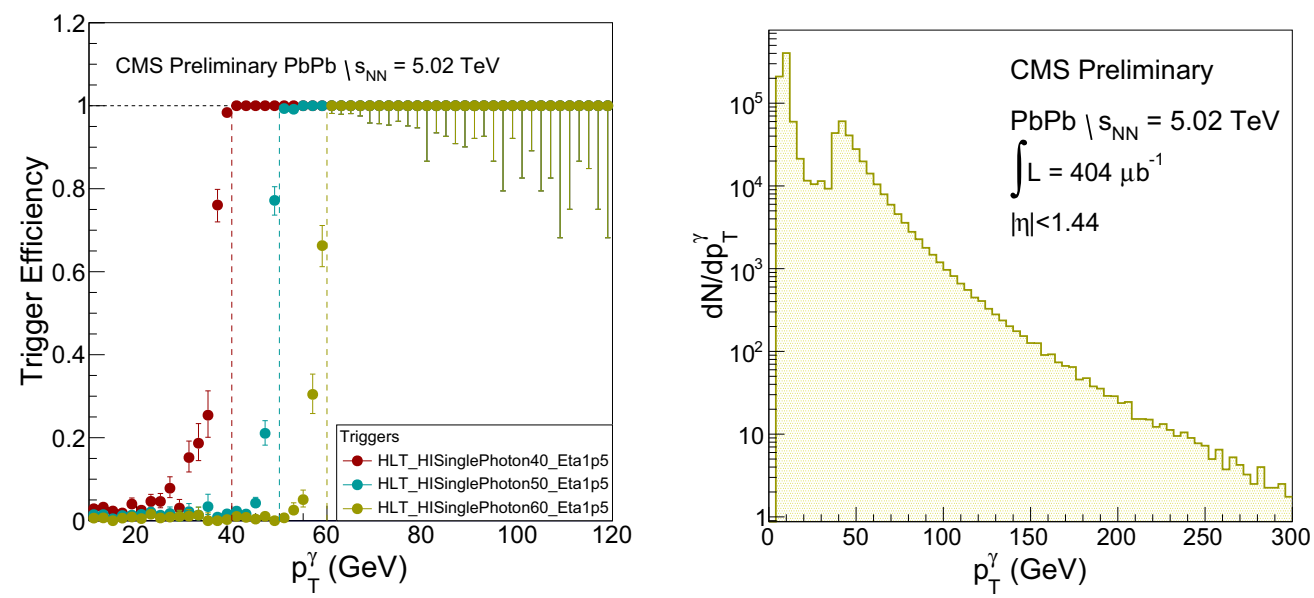

Figure 1. Photon trigger efficiency in $\mathrm{PbPb}$ data as a function of the reconstructed $E_{T}$ (left), computed using a minimum bias data sample. It shows that for photons with an $E_{T}>40 \mathrm{GeV} / \mathrm{c}$ the trigger was nearly $100 \%$ efficient. Reconstructed photon spectra as a function of $p_{T}$ (right) have been measured without isolation condition.

\subsection{Reconstruction Efficiency}

Reconstruction efficiency is the fraction of reconstructed photons in generated photons without isolation condition as shown in Figure 2 left panel. There is no centrality dependence of the reconstruction efficiency for all $p_{T}$ region. Reconstruction efficiency is over $98 \%$ in all centrality and $p_{T}$ region.

\subsection{Isolation Efficiency}

Isolation Efficiency is defined as reconstructed photons passing isolation selection in all reconstructed photons. Table 1 summarizes the isolated photon selections.

Table 1. Isolated photon selection

\begin{tabular}{|c|}
\hline Isolated photon selection \\
\hline $\mathrm{H} / \mathrm{E}<0.1$ \\
SumIso $<1 \mathrm{GeV}$ \\
$\sigma_{\text {ini }}<0.01$ \\
\hline
\end{tabular}

The $H / E$ ratio is defined as the fraction of hadronic energy to the electromagnetic energy in an angular region of cone radius $R=\sqrt{(\Delta \eta)^{2}+(\Delta \phi)^{2}}=0.15$ which is computed from the energy deposition in ECAL and HCAL. This variable is used to reject candidates originating from jets, which carry not only electromagnetic energy but also hadronic energy. In this analysis, the $H / E$ ratio of the photon candidates is required to be smaller than 0.1 in order to reject candidates originating from jets.

Isolation variable 'SumIso' is defined as energy sum in a $R=\sqrt{(\Delta \eta)^{2}+(\Delta \phi)^{2}}=0.4$ cone around photon candidates, which is tracker $E_{T}+$ ecal $E_{T}+$ hcal $E_{T}$. SumIso condition selects isolated photons. 

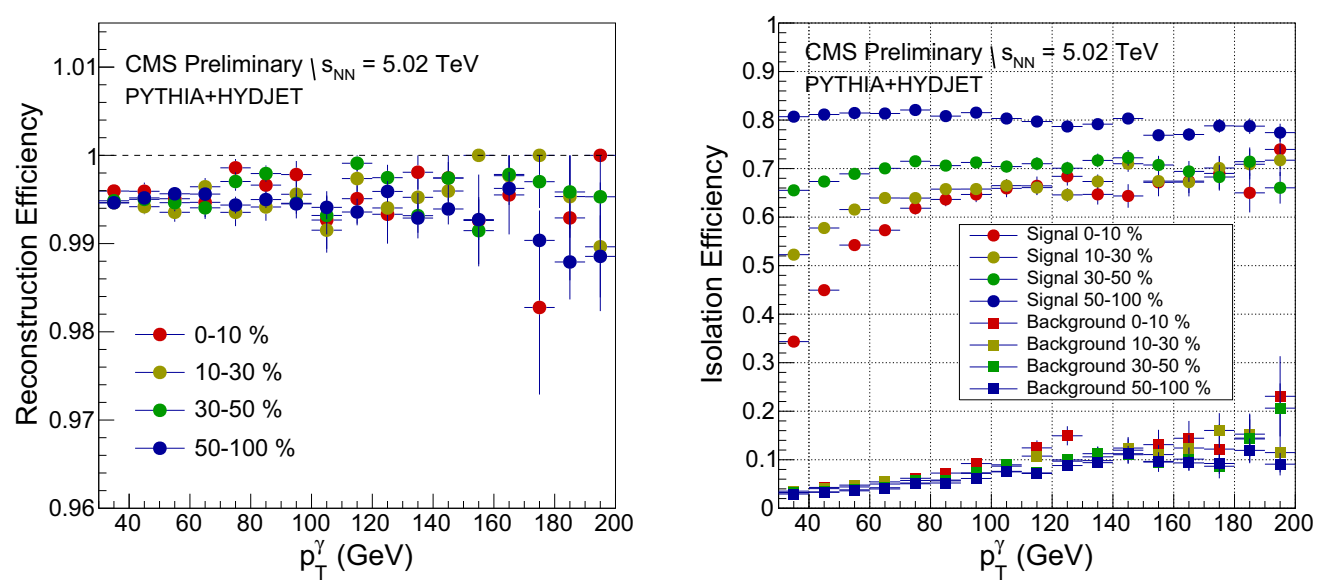

Figure 2. Reconstruction efficiency (left) and isolation efficiency (right) as a function of reconstructed photon $p_{T}$ based on PYTHIA+HYDJET samples. Different colors represent different centrality range.

The $\sigma_{\text {ini }}$ characterizes the shower shape in the $\eta$ direction which is defined as:

$$
\sigma_{i \eta \eta}^{2}=\frac{\sum_{i}^{5 \times 5} w_{i}\left(\eta_{i}-\eta_{5 \times 5}\right)^{2}}{\sum_{i}^{5 \times 5} w_{i}}, w_{i}=\max \left(0, c+\ln \frac{E_{i}}{E_{5 \times 5}}\right),
$$

where $E_{i}$ and $\eta_{i}$ are the energy and pseudorapidity of the $i^{\text {th }}$ crystal within the $5 \times 5$ electromagnetic cluster. $\sigma_{i \eta i \eta}$ is calculated with logarithmic weights $w_{i} . E_{5 \times 5}$ and $\eta_{5 \times 5}$ are the energy and averaged $\eta$ of the $5 \times 5$ crystals. The value of $c$ is a constant which was set to 4.7 in the pp studies and is effectively a cutoff on the crystal energy. The isolated photon candidates tend to have smaller $\sigma_{i \eta i}$ while hadrons and decay photon from $\pi^{0}$ s tend to have larger $\sigma_{i \eta i \eta}$. [1]

Isolation efficiencies for the signal and the background are shown in Figure 2 right panel for different centrality bins. Most of the background photons are rejected by isolation condition according to photon $p_{T}$. These background photons are mainly decay photons from neutral meson such as $\pi^{0}$ and $\eta$ and also non-isolated prompt and fragmentation photons such as jets. Isolation efficiency for the signal depends on centrality. It decreases with increasing centrality and decreasing $p_{T}$. But, isolation efficiency for the background has no strong dependence on the centrality.

\section{Purity}

The selection criteria described above yield a relatively pure sample of isolated photons. Yet, there are still non-prompt photons, such as those from an isolated $\pi^{0}$ carrying a large fraction of the parent fragmenting parton energy, which can pass the isolation cuts. The fraction of those remaining backgrounds is statistically calculated using a two-component fit of the shape of the electromagnetic shower in ECAL $\left(\sigma_{i \eta \eta}\right)$, called the Template method.

A template is defined as the probability distribution function of $\sigma_{i \eta i}$ of either pure photons (signal template) or pure background (e.g. $\pi^{0}, \eta$ ) (background template). The signal template was obtained from MC photon samples (PYTHIA+HYDJET for PbPb or PYTHIA for $\mathrm{pp}$ ), and the background template 


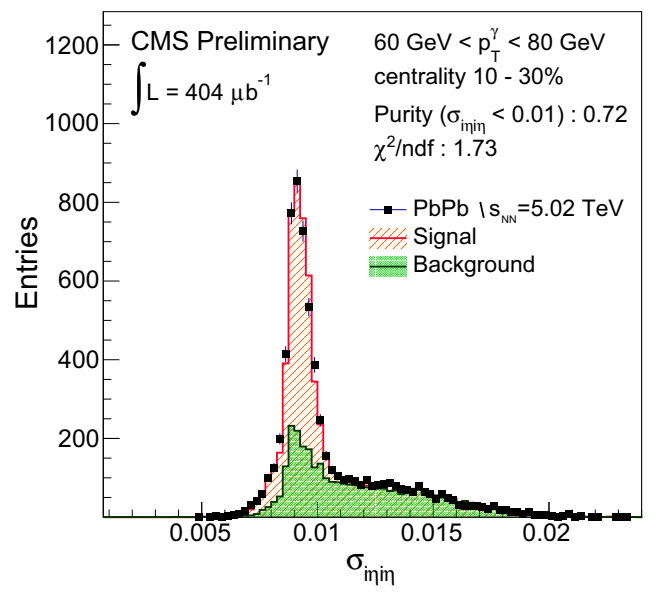

Figure 3. The shower shape variable $\left(\sigma_{i \eta \eta \eta}\right)$ of photons from mid-central $(10-30 \%) \mathrm{PbPb}$ collisions. The black points are $\mathrm{PbPb}$ data, the red histogram is the signal template from PYTHIA+HYDJET, and the green histogram is the background template from a non-isolated data sideband. The purity is defined as the fraction of isolated photons in the signal region $\sigma_{i \eta i \eta}<0.01$.

was obtained from data in a sumIso sideband $(10 \mathrm{GeV}<$ SumIso $<20 \mathrm{GeV})$ where the background is enriched. The yield of signal photons is estimated with a binned maximum likelihood fit to the $\sigma_{i \eta i}$ distribution of data with the signal and background templates. The purity is defined as the fraction of photons with $\sigma_{\text {ini }}<0.01$ (chosen to maximize the purity value) which are accounted for by the signal template.[1]

An example of the template fitting method is shown in Figure 3.

\section{Summary}

Reconstruction and identification of isolated photons have been developed in heavy ion collisions from the data collected during run 2 CMS experiment and PYTHIA+HYDJET Monte-carlo simulation. Trigger, reconstruction and isolation efficiencies are presented and purity value using data-driven method is observed. An isolation condition is optimized to maximize the yield of prompt photon and reject the background photons from neutral meson decay. Isolated photon measurement will give a better understanding of the strongly interacting medium in heavy ion collisions.

\section{References}

[1] CMS collaboration, "Photon-Jet correlations in $\mathrm{PbPb}$ and pp collisions at center of mass energy 5.02 TeV", CMS Physics Analysis Summary CMS-PAS-HIN-16-002, CERN (2016) 